\title{
A Bank of Reconfigurable LQG Controllers for Linear Systems Subjected to Failures ${ }^{1}$
}

\author{
S. Kanev, M. Verhaegen \\ Faculty of Applied Physics, \\ University of Twente, \\ P.O. Box 217, 7500 AE Enschede, The Netherlands \\ tel. +31534892882, +31534893181 \\ S.K.Kanev@TN.UTwente.NL and M.Verhaegen@TN.UTwente.NL
}

\begin{abstract}
In this paper an approach for controller reconfiguration is presented. The starting point in the analysis is a sufficiently accurate continuous linear time-invariant (LTI) model of the nominal system. Based on a bank of reconfigurable LQG controllers, each designed for a particular combination of total faults, the reconfiguration consists in two operation modes. In the first mode a switching is invoked towards one of the pre-designed LQG controllers on the basis of the information about only the combination of total faults that is in effect. In the second mode, which is activated in cases of partial and component faults, a dynamic correction procedure is initiated which tries to reconfigure the currently active controller in such a way, that the failed closed-loop system remains stable and its performance is as close as possible to the performance of the closed-loop system with only total faults present in the system. In cases of partial faults the second mode is practically an extension of the modified pseudo-inverse method. In cases of component faults the second mode is based on an LMI optimization problem. The approach is illustrated using a model of a real-life space robot manipulator, in which total, partial and component faults are simulated.
\end{abstract}

Keywords: Reconfigurable control, Fault-tolerant systems, Multiple-Model Control, LMI.

\section{Introduction}

The increasing complexity of modern control systems require their ability for operation over long periods of time, without human intervention. These systems should possess the capability to accommodate faults, i.e. they should be fault-tolerant. Typical examples of such systems are systems that operate in space $[3,9,16]$ since they have predefined scheduled tasks, requiring timely completion. Other

\footnotetext{
${ }^{1}$ This work was sponsored by the Dutch Technology Foundation (STW) under project number DEL. 4506.
}

examples are aircraft systems $[15,5,1,2]$ which will in the coming future operate autonomously with the requirement of highly increased survivability. The future fully automated traffic control (FATC) [10] having the purpose to reduce the human involvement in highway traffic as much as possible, is yet another example of systems in which the issue of fault accommodation is of critical importance.

The problem of reconfigurable control has been addressed in the literature in different forms. In general, the following categories can be distinguished: multiple model adaptive control $[1,13,9]$, the pseudo-inverse method $[7,14]$, adaptive control [2], predictive control [11, 12], controlallocation reconfiguration [5], eigenstructure assignment [17], input-output linearization [6]. However, most of the existing techniques are either based on the assumption that the possible system faults are known a priori, i.e. only anticipated faults are "allowed", or consider only a given class of the possible system faults. The current paper aims at the development a controller reconfiguration (CR) methodology allowing stabilizing control in all cases of (sensor, actuator and component) faults, under which the systems remains stabilizable and detectable. By sensor and actuator faults we mean linear faults that can be modeled by re-scaling of the inputs and outputs of the system. By component fault a deviation of a physical parameter of the system from its nominal value is meant.

A fault-tolerant system (FTS) in general consists of two main parts. The first part is a fault detection and diagnosis (FDD) mechanism [8], having the task to detect faults in the system as well as to distinguish the origin and the magnitude of the detected faults (diagnosis). The-second part is controller reconfiguration which aims at maintaining a satisfactory level of closed-loop performance in cases of faults in the system. This paper focuses on the CR part of the FTS system. The information about faults is assumed to be precise and timely, supplied by the FDD part of the faulttolerant control system. Nowadays, a lot of different types of algorithms for fault detection and diagnosis exist, some of which could be interconnected with the proposed technique to yield a complete fault-tolerant system. Readers who are 
interested in schemes for FDD are referred to [8] and the references therein.

In general, given a linear system, the optimal performance of the nominal closed-loop system can always be preserved when the system undergoes only partial faults in its sensors and actuators. Then the CR simply consists of inputoutput scaling. However, this performance could not, in general, be achieved when the system experiences total (sensor and actuator) or component faults. Thus, it is desirable to have different performance objectives for the different total and component faults. Unfortunately, the set of all possible component faults is not finite, which makes it impossible and impractical to construct different performance criteria for them. There is, however, a finite number of possible total sensor and total actuator faults. Therefore, we could attribute a different cost function to the different combinations of total faults, and we could design an optimal LQG controller for each model that represents such a combination of total faults. Then, whenever a total fault occurs we only need to activate the corresponding controller. Given an active controller we could try to reconfigure it in the case a partial or a component fault occurs.

The paper is organized as follows. The next section introduces the model representation of faults that is assumed throughout the paper. Section 3 presents the main idea behind the proposed algorithm for controller reconfiguration, which guarantees stability in cases of (both partial and total) sensor and actuator faults. In section 4 the algorithm is extended to assure the stability in cases of component faults as well. The proposed methodology is illustrated in section 5 by a case study with a real-life space robot manipulator, in which different faults are simulated. Finally, section 6 presents the conclusions, and section 7 - the acknowledgments.

\section{Modeling Sensor and Actuator Failures}

The notation is standard. $R \mathcal{H}_{\infty}$ will denote the space of real rational stable transfer matrices. The pseudo-inverse of a matrix $A$ is denoted as $A^{\dagger} . \mathcal{F}_{L}(\bullet, \bullet)$ will represent the lower linear fractional transformation (LFT). The transfer matrix of a system will be denoted

$$
S=\left[\begin{array}{c|c}
A & B \\
\hline C & D
\end{array}\right]=C(s I-A)^{-1} B+D .
$$

Consider the nominal model of the system:

$$
S:\left\{\begin{array}{l}
\dot{x}(t)=A x(t)+B u(t)+T \xi(t) \\
y(t)=C x(t)+D u(t)+\eta(t)
\end{array}\right.
$$

where $x(t) \in \mathbb{R}^{n}$ is the system state, $u(t) \in \mathbb{R}^{m}$ is the input to the system, $y(t) \in \mathbb{R}^{p}$ is the output of the system. $\xi \in \mathbb{R}^{n_{\xi}}$ and $\eta \in \mathbb{R}^{p}$ are process and measurement noises with means $\bar{\xi}$ and $\bar{\eta}$, and covariances $V$ and $W$, respectively. Since the algorithm developed in this paper is based on LQG control, which has limited robustness properties, it is assumed that a sufficiently accurate nominal model of the system is given.

In what follows we will model each actuator (sensor) fault, either total or partial, by multiplication of the corresponding column (row) of the nominal $B(C)$ matrix by a positive scalar in the interval $[0,1)$, where zero corresponds to total fault.

Thus, if we define the set

$$
\Sigma^{r}=\left\{\Sigma: \Sigma=\operatorname{diag}\left\{\sigma_{1}, \ldots, \sigma_{r}\right\}, \sigma_{i} \in \mathbb{R}, \sigma_{i} \in[0,1], i=1, \ldots, r\right\}
$$

then all possible sensor and actuator faults can be modeled as

$$
S_{f}:\left\{\begin{array}{l}
\dot{x}_{f}(t)=A x_{f}(t)+B \Sigma_{a} u(t)+T_{i} \xi(t) \\
y_{f}(t)=\Sigma_{s} C x_{f}(t)+\Sigma_{s} D \Sigma_{a} u(t)+\eta(t)
\end{array}\right.
$$

with $\Sigma_{a} \in \Sigma^{m}$, and $\Sigma_{s} \in \Sigma^{p}$. In the case when $\xi(t)$ is additive to the input $u(t)$, we have $T=B$ and $T_{i}=B \Sigma_{a}$.

We will make the assumption that the pair $\left(A, B \Sigma_{a}\right)$ is stabilizable, and the pair $\left(A, \Sigma_{s} C\right)$ is detectable. Otherwise, there exists no controller that can stabilize the closed-loop system.

In general, models representing component faults cannot be described in such a convenient form. Depending on the location of the failed component, it could affect any one of the system $(A, B, C, D)$ matrices.

\section{A Bank of Reconfigurable LQG Controllers}

The controller reconfiguration approach that will be presented here consists of a bank of LQG controllers, each corresponding to a particular combination of total (sensor and actuator) faults (figure 1). Then, when a total fault (or a combination of total faults) takes place, the controller that is designed to be optimal for the current model of the failed system is activated. If afterwards some partial fault occurs, the transfer matrix of the currently active controller is preand post-multiplied by two gain matrices so that the optimal performance of the closed-loop system, that was active before the occurrence of this partial fault, is preserved. Thus, no performance degradation of the closed-loop system can be expected as a result of (both sensor and actuator) partial faults.

However, the situation is different in case of component faults. Such faults may, in general, lead to changes in all of the matrices in the state space model. Whenever a component fault occurs our primary goal will be to assure the stability of the closed-loop system. Once the stability requirement is fulfilled, we will try to reconfigure the controller in such a way, that the resulting closed-loop system is as close as possible (in some sense) to the closed-loop system before the occurrence of the component fault. 


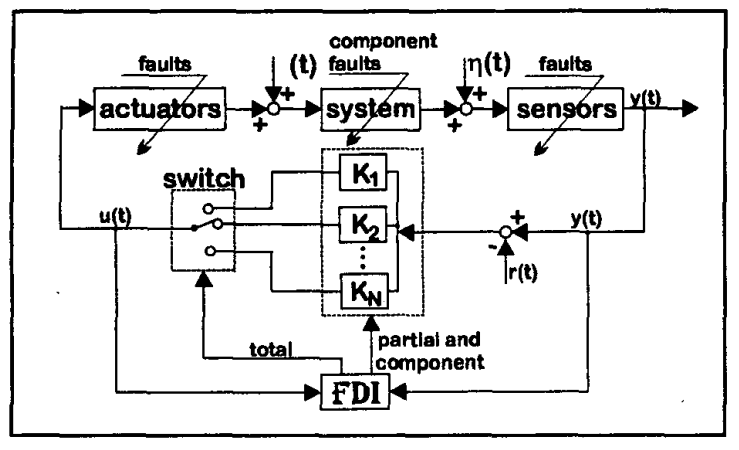

Figure 1: Interconnection of the bank of reconfigurable LQG controllers with the other components of the fault-tolerant system.

Let $N$ be the number of possible combinations of total faults for which the system is still stabilizable and detectable. We design an LQG controller for each model that represents one of these combinations of total faults. The number of controllers in the bank, in general, is $N \leq 2^{m+p}-2^{m}-2^{p}+1$. Thus, we have a set of $N$ cost functions

$$
J_{i}=\int_{0}^{\infty}\left[x_{i}^{T}(t) u^{T}(t)\right]\left[\begin{array}{cc}
Q_{i} & 0 \\
0 & R_{i}
\end{array}\right]\left[\begin{array}{l}
x_{i}(t) \\
u(t)
\end{array}\right] d t
$$

with $Q_{i} \geq 0$ and $R_{i}>0, \forall i=1, \ldots, N$, and a bank of $N$ optimal LQG controllers $K_{i}(s)$, each optimizing its corresponding cost function $J_{i}$.

Now, consider the transfer matrix of the model that corresponds to the $i$-th combination of total faults

$$
G_{i}(s)=\left[\begin{array}{c|c}
A & B_{i} \\
\hline C_{i} & D_{i}
\end{array}\right],
$$

with state vector $x_{i} \in \mathbb{R}^{n}$.

Given this model of total faults, any model representing a subsequent occurrence of partial faults will be denoted by an upper suffix "P" $\left(G_{i}^{P}\right)$, while models for occurrences of component faults - by a bar $\left(\bar{G}_{i}\right)$. If both partial and component faults occur, the model will be denoted as

$$
\bar{G}_{i}^{P}=\left[\begin{array}{c|c}
\bar{A} & \bar{B}_{i}^{P} \\
\hline \bar{C}_{i}^{P} & \bar{D}_{i}^{P}
\end{array}\right]=\left[\begin{array}{c|c}
\bar{A} & \bar{B} \Sigma_{i, a} \\
\hline \Sigma_{i, s} \bar{C} & \Sigma_{i, s} \bar{D} \Sigma_{i, a}
\end{array}\right],
$$

The corresponding LQG controller for the model $G_{i}(s)$ is

$$
K_{i}(s)=\left[\begin{array}{c|c}
A+B_{i} F_{i}+L_{i} C_{i}+L_{i} D_{i} F_{i} & -L_{i} \\
\hline F_{i} & 0
\end{array}\right] .
$$

where $x_{C, i} \in \mathbb{R}^{n}$ is its state vector, $F_{i}=R_{i}^{-1} B_{i}^{T} X_{i}$ is the state feedback gain with $X_{i}$ the solution of the Riccati equation

$$
A^{T} X_{i}+X_{i} A-X_{i} B_{i} R_{i}^{-1} B_{i}^{T} X_{i}+Q_{i}=0
$$

and $L_{i}=Y_{i} C_{i}^{T} V^{-1}$ is the Kalman filter gain with $Y_{i}$ - the solution of the Riccati equation

$$
A Y_{i}+Y_{i} A^{T}-Y_{i} C_{i}^{T} V^{-1} C_{i} Y_{i}+W=0 .
$$

Then the autonomous part of the closed-loop system is described as

$$
\begin{aligned}
\mathcal{F}_{L}\left(G_{i}, K_{i}\right):\left\{\left[\begin{array}{c}
\dot{x}_{i}(t) \\
\dot{x}_{C, i}(t)
\end{array}\right]=\right. \\
{\left[\begin{array}{cc}
A & B_{i} F_{i} \\
-L_{i} C_{i} & A+B_{i} F_{i}+L_{i} C_{i}
\end{array}\right]\left[\begin{array}{c}
x_{i}(t) \\
x_{C, i}(t)
\end{array}\right] . }
\end{aligned}
$$

We want to reconfigure the controller $K_{i}(s)$ in the case when some partial faults of the remaining sensors and actuators, or some component faults, occur. The information on the present total and partial faults will be represented by the diagonal elements of the two matrices $\Sigma_{i, a} \in \Sigma^{m}$ and $\Sigma_{i, s} \in \Sigma^{p}$, as explained in section 2. Note that $B_{i}^{P}=B \Sigma_{i, a}=B_{i} \Sigma_{i, a}$, $C_{i}^{P}=\Sigma_{i, s} C=\Sigma_{i, s} C_{i}$, and $D_{i}^{P}=\Sigma_{i, s} D \Sigma_{i, a}=\Sigma_{i, s} D_{i} \Sigma_{i, a}$. Thus, given the model $\left(A, B_{i}, C_{i}, D_{i}\right)$ that corresponds to the $i$-th combination of total sensor and actuator faults, each additional possible partial and component fault will be represented by a model with state-space matrices

$$
\left(\bar{A}, \bar{B}_{i}^{P}, \bar{C}_{i}^{P}, \bar{D}_{i}^{P}\right)=\left(\bar{A}, \bar{B} \Sigma_{i, a}, \Sigma_{i, s} \bar{C}, \Sigma_{i, s} \bar{D} \Sigma_{i, a}\right) .
$$

If we write the corresponding controller of this system as

$$
\bar{K}_{i}^{P}(s)=\left[\begin{array}{c|c}
\bar{A}+\bar{B}_{i}^{P} \bar{F}_{i}+\bar{L}_{i} \bar{C}_{i}^{P}+\bar{L}_{i} \bar{D}_{i}^{P} \bar{F}_{i} & -\bar{L}_{i} \\
\hline \bar{F}_{i} & 0
\end{array}\right],
$$

then, the autonomous part of the closed-loop system becomes

$$
\begin{aligned}
& \mathcal{F}_{L}\left(\bar{G}_{i}^{P}, \bar{K}_{i}^{P}\right):\left\{\left[\begin{array}{c}
\dot{\bar{x}}_{i}(t) \\
\dot{\bar{x}}_{C, i}(t)
\end{array}\right]=\right. \\
& {\left[\begin{array}{cc}
\bar{A} & \bar{B} \Sigma_{i, a} \bar{F}_{i} \\
-\bar{L}_{i} \Sigma_{i, s} \bar{C} & \bar{A}+\bar{B} \Sigma_{i, a} \bar{F}_{i}+\bar{L}_{i} \Sigma_{i, s} \bar{C}
\end{array}\right]\left[\begin{array}{c}
\bar{x}_{i}(t) \\
\bar{x}_{C, i}(t)
\end{array}\right] .}
\end{aligned}
$$

Since the poles of the closed-loop systems (7) and (9) are the eigenvalues of the matrices

$$
\left[\begin{array}{cc}
A+L_{i} C_{i} & 0 \\
0 & A+B_{i} F_{i}
\end{array}\right] \text { and }\left[\begin{array}{cc}
\bar{A}+\bar{L}_{i} \Sigma_{i, s} \bar{C} & 0 \\
0 & \bar{A}+\bar{B} \Sigma_{i, a} \bar{F}_{i}
\end{array}\right]
$$

respectively, our aim will be to find the matrices $\bar{L}_{i}$ and $\bar{F}_{i}$ that solve the optimization problem:

minimize over $\bar{L}_{i}, \bar{F}_{i}$ the function

$$
\left\|\left[\begin{array}{cc}
A+L_{i} C_{i} & 0 \\
0 & A+B_{i} F_{i}
\end{array}\right]-\left[\begin{array}{cc}
\bar{A}+\bar{L}_{i} \Sigma_{i, s} \bar{C} & 0 \\
0 & \bar{A}+\bar{B} \Sigma_{i, a} \bar{F}_{i}
\end{array}\right]\right\|_{F}
$$

Lemma 3.1. The solution of the optimization problem (10) is given by

$$
\begin{aligned}
& \bar{L}_{i}=\left(A-\bar{A}+L_{i} C_{i}\right) \bar{C}^{\dagger} \Sigma_{i, s}^{\dagger}, \\
& \bar{F}_{i}=\Sigma_{i, a}^{\dagger} \bar{B}^{\dagger}\left(A-\bar{A}+B_{i} F_{i}\right)
\end{aligned}
$$

Proof: The optimization problem can be split into two separate optimization problems:

$$
\min _{\bar{L}_{i}} J_{1}=\min _{\bar{L}_{i}}\left\|\left(A+L_{i} C_{i}\right)-\left(\bar{A}+\bar{L}_{i} \Sigma_{i, s} \bar{C}\right)\right\|_{F}
$$


and

$$
\min _{\bar{F}_{i}} J_{2}=\min _{\bar{F}_{i}}\left\|\left(A+B_{i} F_{i}\right)-\left(\bar{A}+\bar{B} \Sigma_{i, a} \bar{F}_{i}\right)\right\|_{F}
$$

Denote

$$
A-\bar{A}+L_{i} C_{i}=\left[\begin{array}{c}
a_{1} \\
\vdots \\
a_{n}
\end{array}\right] \in \mathbb{R}^{n \times n} \text { and } \tilde{L}_{i}=\left[\begin{array}{c}
l_{1} \\
\vdots \\
l_{n}
\end{array}\right] \in \mathbb{R}^{n \times p} \text {. }
$$

Then the first optimization problem can be written as

$$
\min _{\bar{L}_{i}}\left\|\left[\begin{array}{c}
a_{1}-l_{1} \Sigma_{i, s} \bar{C} \\
\vdots \\
a_{n}-l_{n} \Sigma_{i, s} \bar{C}
\end{array}\right]\right\|_{F}^{2}=\min _{\bar{L}_{i}} \sum_{j=1}^{n}\left\|a_{j}-l_{j} \Sigma_{i, s} \bar{C}\right\|_{2}^{2}
$$

Thus, we are looking for the least squares solution of the equation $a_{j}=l_{j} \Sigma_{i, s} \bar{C}$, which is $l_{j}=a_{j} \bar{C}^{\dagger} \Sigma_{i, s}^{\dagger}$. This solution minimizes the vector norm $\left\|a_{j}-l_{j} \Sigma_{i, s} \bar{C}\right\|_{2}^{2}$. Therefore

$$
\bar{L}_{i}=\left[\begin{array}{c}
l_{1} \\
\vdots \\
l_{n}
\end{array}\right]=\left[\begin{array}{c}
a_{1} \\
\vdots \\
a_{n}
\end{array}\right] \bar{C}^{\dagger} \Sigma_{i, s}^{\dagger}=\left(A-\bar{A}+L_{i} C_{i}\right) \bar{C}^{\dagger} \Sigma_{i, s}^{\dagger}
$$

Now, denote

$$
\begin{aligned}
& A-\bar{A}+B_{i} F_{i}=\left[\begin{array}{ccc}
\tilde{a}_{1}, & \cdots, & \tilde{a}_{n}
\end{array}\right] \in \mathbb{R}^{n \times n}, \text { and } \\
& \bar{F}_{i}=\left[\begin{array}{lll}
f_{1}, & \cdots, & f_{n}
\end{array}\right] \in \mathbb{R}^{m \times n} .
\end{aligned}
$$

Then the second optimization problem can be rewritten as

$$
\begin{aligned}
& \min _{\bar{F}_{i}}\left\|\left[\tilde{a}_{1}-\bar{B} \Sigma_{i, a} f_{1}, \cdots, \quad \tilde{a}_{n}-\bar{B} \Sigma_{i, a} f_{n}\right]\right\|_{F}^{2}= \\
& \min _{\bar{F}_{i}} \sum_{j=1}^{n}\left\|\tilde{a}_{j}-\bar{B} \Sigma_{i, a} f_{j}\right\|_{2}^{2}
\end{aligned}
$$

Now, $f_{j}=\Sigma_{i, a}^{\dagger} \bar{B}^{\dagger} \tilde{a}_{j}$ minimizes $\left\|\tilde{a}_{j}-\bar{B} \Sigma_{i, a} f_{j}\right\|_{2}^{2}$ and

$$
\begin{aligned}
& \bar{F}_{i}=\left[f_{1}, \cdots, f_{n}\right] \Sigma_{i, a}^{\dagger} \bar{B}^{\dagger}\left[\tilde{a}_{1}, \cdots, \tilde{a}_{n}\right]= \\
& =\Sigma_{i, a}^{\dagger} \bar{B}^{\dagger}\left(A-\bar{A}+B_{i} F_{i}\right)
\end{aligned}
$$

which completes the proof.

Remark 3.1. When no component faults take place, the algorithm guarantees $\min \left(J_{1}\right)=\min \left(J_{2}\right)=0$, i.e. we have exact matching of the two closed-loop systems when only sensor and actuator faults occur. To see this, note that in this case $\left(\bar{A}, \bar{B}_{i}^{P}, \bar{C}_{i}^{P}, \bar{D}_{i}^{P}\right)=\left(A, B_{i}^{P}, C_{i}^{P}, D_{i}^{P}\right)$. Thus, we have

$$
\mid \begin{aligned}
& \bar{L}_{i}=L_{i} \Sigma_{i, s}^{\dagger} \\
& \bar{F}_{i}=\Sigma_{i, a}^{\dagger} F_{i}
\end{aligned}
$$

If we now substitute these in (10) we get

$$
\left\{\begin{array}{cc}
J_{1} & 0 \\
0 & J_{2}
\end{array}\right]=\text { }\left[\begin{array}{cc}
A+L_{i} C_{i} & 0 \\
0 & A+B_{i} F_{i}
\end{array}\right]-\left[\begin{array}{cc}
A+L_{i} \Sigma_{i, s}^{\dagger} \Sigma_{i, s} C & 0 \\
0 & A+B \Sigma_{i, a} \Sigma_{i, a}^{+} F_{i}
\end{array}\right] \|_{F}
$$

Since $\Sigma_{i, s}^{\dagger} \Sigma_{i, s}$ models only the total sensor faults, and $\Sigma_{i, a} \Sigma_{i, a}^{\dagger}$ models only the total actuator faults, we can write

$$
\mid \begin{aligned}
& \Sigma_{i, s}^{\dagger} \Sigma_{i, s} C=C_{i} \\
& B \Sigma_{i, a} \Sigma_{i, a}^{\dagger}=B_{i}
\end{aligned}
$$

which makes

$$
\left[\begin{array}{cc}
J_{1} & 0 \\
0 & J_{2}
\end{array}\right]=0
$$

Unfortunately, in the case when component faults are present, the matrices $\bar{F}_{i}$ and $\bar{L}_{i}$ do not assure closed-loop stability in general. The section that follows presents a possible solution of this problem by trying to do the same matching while maintaining the stability of the closed-loop system.

\section{Assuring Stability in Cases of Component Failures}

For the case of component faults, the idea is again to solve (10), but now under the constraint that the system (9) is stable. In other words, our goal in cases of component faults will be to find the matrices $\bar{L}_{i}$ and $\bar{F}_{i}$ that solve the following two constraint optimization problems

$$
\mid \begin{gathered}
\min _{\bar{L}_{i}}\left\|\left(A+L_{i} C_{i}\right)-\left(\bar{A}+\bar{L}_{i} \Sigma_{i, s} \bar{C}\right)\right\|_{2} \\
\text { subject to }\left(\bar{A}+\bar{L}_{i} \Sigma_{i, s} \bar{C}\right) \in R \mathcal{H}_{\infty}
\end{gathered}
$$

and

$$
\mid \begin{aligned}
& \min _{\bar{F}_{i}}\left\|\left(A+B_{i} F_{i}\right)-\left(\bar{A}+\bar{B} \Sigma_{i, a} \bar{F}_{i}\right)\right\|_{2} \\
& \text { subject to }\left(\bar{A}+\bar{B} \Sigma_{i, a} \bar{F}_{i}\right) \in R \mathcal{H}_{\infty}
\end{aligned}
$$

One way to solve these problems is to represent them as linear objective minimization problems subject to LMI constraints. For this purpose, let us first note that the stability conditions above can be rewritten as

$$
\mid \begin{aligned}
\exists X_{1}= & X_{1}^{T}>0, \text { and } \bar{L}_{i}, \text { such that } \\
& X_{1}\left(\bar{A}+\bar{L}_{i} \Sigma_{i, s} \bar{C}\right)+\left(\bar{A}+\bar{L}_{i} \Sigma_{i, s} \bar{C}\right)^{T} X_{1}<0 \\
\exists X_{2}= & X_{2}^{T}>0, \text { and } \bar{F}_{i}, \text { such that } \\
& \left(\bar{A}+\bar{B} \Sigma_{i, a} \bar{F}_{i}\right) X_{2}+X_{2}\left(\bar{A}+\bar{B} \Sigma_{i, a} \bar{F}_{i}\right)^{T}<0
\end{aligned}
$$

or, equivalently, by substituting $Y_{1}=X_{1} \bar{L}_{i}$ and $Y_{2}=\bar{F}_{i} X_{2}$, as

$$
\mid \begin{aligned}
\exists X_{1}= & X_{1}^{T}>0, \text { and } Y_{1}, \text { such that } \\
& X_{1} \bar{A}+\bar{A}^{T} X_{1}+Y_{1} \Sigma_{i, s} \bar{C}+\bar{C}^{T} \Sigma_{i, s} Y_{1}^{T}<0 \\
\exists X_{2}= & X_{2}^{T}>0, \text { and } Y_{2}, \text { such that } \\
& \bar{A} X_{2}+X_{2} \bar{A}^{T}+\bar{B} \Sigma_{i, a} Y_{2}+Y_{2}^{T} \Sigma_{i, a} \bar{B}^{T}<0
\end{aligned}
$$

Let us first observe that, in general, the unconstrained minimization problem

$$
\min _{x}\|A x-b\|_{2}
$$

with $A \in \mathbb{R}^{n \times n}, x \in \mathbb{R}^{n}$, and $b \in \mathbb{R}^{n}$, can be restated as an LMI optimization problem the following way [4]

$$
\left[\begin{array}{lc}
\min _{x, t} t \text { subject to: } \\
{[I} & A x-b \\
(A x-b)^{r} & t
\end{array}\right] \geq 0
$$




\begin{tabular}{||l|l||l||}
\hline \hline Parameter: & Symbol: & Value: \\
\hline \hline gearbox ratio & $N$ & -260.6 \\
\hline motor torque constant & $K_{t}$ & 0.6 \\
\hline the damping coefficient & $\beta$ & 0.4 \\
\hline inertia of the input axis & $I_{m}$ & 0.0011 \\
\hline inertia of the output system & $I_{\text {son }}$ & 400 \\
\hline spring constant & $c$ & 130000 \\
\hline \hline
\end{tabular}

Table 1: The nominal values of the parameters in the linear model of one joint of the SRM.

Thus, substituting $\mathcal{A}_{1}=A-\bar{A}+L_{i} C_{i}$ and $\mathcal{A}_{2}=A-\bar{A}+B_{i} F_{i}$, the initial two optimization problems (12) and (13) can be rewritten as

$$
\left.\mid \begin{array}{cccc}
\min _{X_{1}, Y_{1}} t_{1} \text { subject to: } & \\
\boldsymbol{A}_{1} I_{n} & X_{1} \mathcal{A}_{1}-Y_{1} \Sigma_{i s} \bar{C} & 0 & 0 \\
\mathcal{A}_{1}^{T} X_{1}+\bar{C}^{T} \Sigma_{i s} Y_{1 .}^{T} & t_{1} I_{n} & 0 & 0 \\
0 & 0 & d_{1} & 0 \\
0 & 0 & 0 & X_{1}
\end{array}\right]>0
$$

where $d_{1}=-\left(X_{1} \vec{A}+\bar{A}^{T} X_{1}+Y_{1} \Sigma_{i s} \bar{C}+\bar{C}^{T} \Sigma_{i s} Y_{1}^{T}\right)$, and

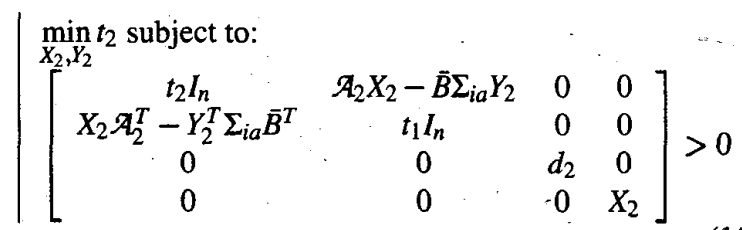

where $d_{2}=-\left(\bar{A} X_{2}+X_{2} \bar{A}^{T}+\bar{B} \Sigma_{i a} Y_{2}+Y_{2} \Sigma_{i a} \bar{B}\right)$.

Given the optimal solutions $X_{1}^{*}, X_{2}^{*}, Y_{1}^{*}$, and $Y_{2}^{*}$, the reconfigured controller (8) is parametrized by

$$
\mid \begin{aligned}
& \bar{L}_{i}=\left(X_{1}^{*}\right)^{-1} Y_{1} \\
& \bar{F}_{i}=Y_{2}\left(X_{2}^{*}\right)^{-1}
\end{aligned}
$$

\section{A Case Study}

In this-section we are going to present the result of a case study, in which we consider a linear model of one joint of a real-life space robot manipulator ([3]). Its state-space representation is given by

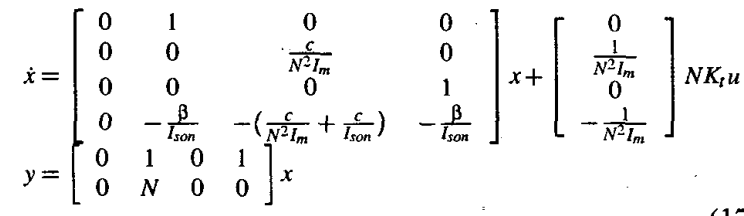

where the nominal values of the physical parameters are given in table 1.

In this experiment the bank of controllers consists of only two controllers: one for the nominal system, and one for a model representing a total fault of sensor No. 2. With this total fault the system is still controllable. It is also assumed that the necessary information from the FDD part is available, exact and without delay.

In this case study we have the following scenario:

a) Models in effect

- for $t \in(0,30]$ the nominal model is in effect, i.e. $\Sigma_{1, s}=I_{2}$, and $\Sigma_{1, a}=1$.

- for $t \in(30,60]$ a faulty model, corresponding to simultaneous total fault of sensor No.2 and partial $40 \%$ fault of sensor No.1, i.e. $\Sigma_{2, s}=\left[\begin{array}{cc}0.4 & 0 \\ 0 & 0\end{array}\right]$, and $\Sigma_{1, a}=1$.

- for $t \in(60,100]$ a component fault $\left(N_{f}=2 . N=\right.$ -521.2) takes place in addition to the total fault of sensor No.2 and the partial $40 \%$ fault sensor No.1. faults

b) Reference Signal

- for $t \in(0,40], r(t)=[1,0]^{T}$.

- for $t \in(40,70], r(t)=[-1,0]^{T}$.

- for $t \in(70,100], r(t)=[-2,0]^{T}$.

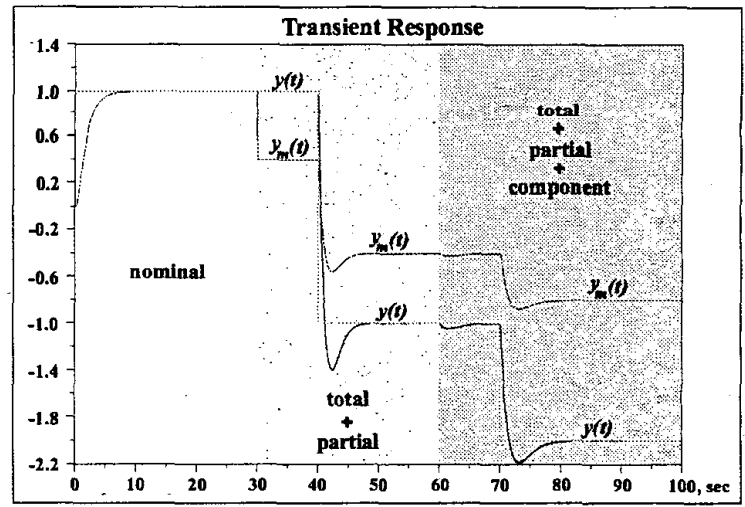

Figure 2: The reference $r(t)$, the measured output $y_{m}(t)$, and the "real" output $y(t)$

In the time interval $t \in(30,60]$ no component faults are active. Then (see Remark 3.1 the reconfigured closed-loop system exactly matches the closed-loop system with only the total fault of sensor No. 2 active. However, for $t>60$ a component fault occurs. With this component fault the reconfiguration process used equation (16) to parametrize the controller as the parametrization (11) did not stabilize the close-loop system.

Figure 2 shows the faulty measured output, the "real" output, and the reference signal. The figure depicts the measurement of the first output, denoted $y_{m}(t)$, the "real" value 
of the first output, denoted $y(t)$, and the reference signal, plotted in dotted line. We could see from the figure the ability of the system to swiftly reconfigure the controller appropriately after each fault occurrence so that the fault is accommodated.

\section{Conclusions}

In this paper we proposed a multiple-model based approach for controller reconfiguration. It consists of a bank of LQG controllers, each designed for the system with only (a combination of) total faults present in the system. Switching between controllers is invoked whenever certain combination of total faults occur. Any additional occurrence of partial or component fault leads to reconfiguration of the currently active controller from the bank. The assumption is made that all faults that occur in the system are swiftly and precisely detected and diagnosed by the FDD part of the FTS. In addition, a sufficiently accurate nominal model of the system is required due to the lack of robustness in the LQG controllers. The approach was illustrated by a case study with a linear model of one joint of a real-life space robot manipulator, in which total and partial sensor faults, as well as a (gearbox ratio) component fault, were simulated. The experimental results show fast reconfiguration and perfect reference tracking of the reconfigured system.

\section{Acknowledgments}

The authors would like to thank the Dutch National Aerospace Laboratory (NLR), and especially to Dr. J.F.T. Bos, for providing us with the data for the space robot manipulator.

\section{References}

[1] Athans, M., Castanon, D., Dunn, K., Greene, C., Lee, W., Sandell, N., and Willsky, A. (1977) The Stochastic Control of the F-8C Aircraft Using Multiple Model Adaptive Control (MMAC) Method - Part 1: Equilibrium Flight, IEEE Trans. on Automatic Control, Vol. 22, No. 5, Oct. 1977

[2] Bodson, M., and Groszkiewicz, J. E. (1997) Multivariable Adaptive Control Algorithms for Reconfigurable Flight Control, IEEE Transactions on Control Systems Technology, Vol. 5, No. 2, March, 1997

[3] Boumans, R. (1996) ERA: Baseline Capabilities and Future Perspectives, Proceedings of the 4th ESA Workshop on Advanced Space Technologies for Robotic Applocations ASTRA, Noordwijk, Netherlands, Nov. 1996

[4] Boyd, S., and Vanderberghe, L. (1993) Notes on Interior-Point Methods for Positive Definite Programming, CDC Workshop, San Antinio, 13 December 1993
[5] Burken, J., Lu, P. and Wu, Z. (1999) Reconfigurable Fligth Control Designs with Application to the X-33 Vehicle, On AIAA Guidance Navigation and Control Conference, Portland, Oregon, AIAA-99-4134, August, 1999

[6] Dardinier-Maron, Hamelin, and Noura(1999) A Fault Tolerant Control Design Against Major Actuator Failures: Application to a Three Tank System, Proceedings of the 38th IEEE Conference on Decision and Control, Phoenix, Arizona, Dec. 1999

[7] Gao, Z., and Antsaklis, P. (1991) Stability of the Pseudo-Inverse Method for Reconfigurable Control Systems, International Journal of Control, 1991, vol. 53, No. 3, pp. 717-729

[8] Gertler, J. J. (1998) Fault Detection and Diagnosis in Engineering Systems, Marcel Dekker, 1998

[9] Griffin, G. C., and Meybeck, P. S. (1997) MMAE/MMAC for Bending with Multiple Uncertain Parameters, IEEE Transaction on Aerospace and Electornic Systems, Vol. 33, No. 3, July, 1997

[10] Huang, S., and Ren, W. (1999) Autonomous Intelligent Vehicle and its Performance in Automated Traffic Systems, International Journal of Control, Vol. 72, No. 18, 1999

[11] Huzmezan, M., and Maciejowski, J. M. (1999) Reconfigurable Flight Control During Actuator Failures Using Predictive Control, 14th Triennial World Congress of IFAC, Beijing, P.R. China, 1999

[12] Kanev, S., and Verhaegen, M. (1999) Controller Reconfiguration for Non-Linear Systems, submitted to Control Engineering Practise, Nov. 1999

[13] Meybeck, P. S., and Stevens, R. D. (1991) Reconfigurable Flight Control via Multiple Model Adaptive Control Methods, IEEE Transactions on Aerospace and Electronic Systems, Vol. 27, No. 3; May, 1991

[14] Noura, H., Sauter, D., Hamelin, F, and Theilliol (2000) Fault-Tolerant Control in Dynamic Systems: Application to a Winding Machine. IEEE Control Systems Mag. azine, February, 2000, p.p. 33-49

[15] Rauch, H. E. (1994) Intelligent Fault Diagnosis and Controller Reconfiguration, IEEE International Symposium on Intelligent Control, Chicago, IL, August 1993

[16] Yen, G. G. (1994) Reconfigurable Learning Control in Large Space Structures, it IEEE Transactions on Control Systems Technology, Vol. 2., No. 4, Dec., 1994

[17] Zhang, Y., and Jiang, J. (1999) Design of Integrated Fault Detection, Diagnosis and Reconfigurable Control Systems, Proceedings of the 38th IEEE Conference on Decision and Control, Phoenix, Arizona, Dec. 1999 